addition to the quota available for ordinary use to the distributor. Only they forgot that an unscrupulous publican can inhibit all ordinary sales and provide distribution only by provision of a medical certificate for same, and as his supply of the latter is reimbursed for each medical certificate provided, the more medical certificates he receives the greater facility he is provided with to dispose of his weekly quota at gratifying prices to himself. That honest hostelers do exist, distributing their quota in fair portion to all their customers while still reserving a small percentage for immediate essential medical use, rather proves that the original reservation is not essential, nor in fact desirable.

This allegorical approach enables me to state with conviction that I have never seen half a valuable page of the Journal put to a greater degree of useless blether than is contained in the Supplement of March 15 (p. 33) describing what is apparently a face-saving measure for the original restrictions imposed on such an essential female garment as a corset, such measure in any case still stimulating the corsetière to obtain a quota-free corset for her client in preference to the private made-tomeasure article, as the former is still apparently available free of purchase tax.

I note these arrangements have not been drawn up in consultation with the manufacturers, and venture to suggest that their advice might have been taken as to how they have successfully surmounted the prolonged epidemic of "severe visceroptosis" despite their shortage of skilled labour. The matter undoubtedly may now be left to their initiative in the confidence that essential demands will be met and the initiation of visceroptotic anxiety left to the hands of the charlatan.I am, etc.,

Tipton, Staffs.

L. H. Eunson.

\section{Diet and Canine Hysteria}

SIR,- Sir Edward Mellanby's notion that canine hysteria was produced by feeding growing dogs on agenized flour (Dec. 14, 1946 , p. 885) cannot be reconciled with experience of the behaviour of healthy dogs or the pathology of those diseased dogs which exhibit canine hysteria. It is very difficult to assess Sir Edward's data, since he omitted to correlate his clinical observations with the ante- and post-mortem pathology of his laboratory dogs and rats. Moreover, his statement that fits were observed "in the animals receiving treated [agenized] flour and were never seen in those having untreated flour" was qualified by his earlier statement that fits "could often be produced" by feeding agenized flour. [my italics].

It would seem that some of the dogs which were fed on agenized flour did not show fits, but I cannot find any description of them: they are not illustrated in Sir Edward's three tables; they obtained even less notice than the rats, which were uniformly negative. This evidence should have been detailed since it is consistent with the anomalies of naturally occurring canine hysteria, of which I will cite two examples from my own records:

(1) No further fits have been observed in the dogs whose diet has been changed from biscuit to meat, whereas fits have persisted in some of the dogs whose diet (biscuit) has been maintained. Some observers have been misled by this phenomenon into attributing the cause of canine hysteria to certain dog biscuits. But I have studied canine hysteria in dogs which, since weaning, had been fed exclusively on meat. (2) No further fits have been observed in the dogs from which roundworms have been expelled, whereas fits have persisted in some of the untreated dogs, although no change has been made in the diet. Many have been influenced by this phenomenon into attributing canine hysteria solely to roundworms. For an understanding of the clinical phenomena the pathology must be fully studied.

My observations of naturally occurring canine hysteria have been incorporated in papers on the B.H.S. infection, which is a widely adopted abbreviation for the infection by various Lancefield groups of the $\beta$-haemolytic streptococcus (Hare and Fry, 1938 ; Hare, 1946). Canine hysteria is not a disease entity it is an event which may occur in the course of a particular infection; it is a type of spasmophilia which is expressed by a proportion of dogs infected by the B.H.S. and roundworms (Toxascaris sp. and/or Toxocara sp.). The essential pathology of dogs which have been destroyed within 24 hours of showing canine hysteria is that of the B.H.S. infection plus the presence of a variable number of roundworms in the duodenum -i.e., chronic tonsillitis, hyperplasia of the reticulo-endothelial system, catarrh of the pylorus of the stomach and duodenum, albuminous degeneration of the liver and kidneys, and, as first recorded by the late Prof. John Eyre (1928), toxic encephalopathy.

In my cases I have not observed "loss of consciousness" and "epileptiform convulsions," which Sir Edward reported in some of his dogs. The label "epileptiform convulsions" (without loss of consciousness) was applied by nineteenth-century French and British veterinary authors to what is now termed canine hysteria. More recent work on the pathology has clarified the differential diagnosis of at teast four distinct types of spasmophilia of dogs, one of these types being epileptiform convulsions, which are now recognized as due to the B.H.S. infection complicated by coccidiosis. Unfortunately some authors carelessly apply the words "epileptiform convulsions" (with loss of consciousness) to the behaviour of dogs which are in the condition of hepatic and renal failure. This is the pathology of the terminal stage of several diseases, one of which is the conjoint B.H.S. and roundworm infections.

From Sir Edward's statement that " a time may come, however, when a dog which soems to be acquiring immunity suddenly suffers the most severe epileptiform fits and dies "I infer that his dogs, to which he attributed "epileptiform convulsions" and "loss of consciousness," were in a condition of hepatic and renal failure. Sir Edward's description of his dogs' behaviour, apart from "epileptiform convulsions" and "loss of consciousness," seems to me to be consistent with the symptomatology of naturally occurring canine hysteria, which was described in French and British veterinary literature of over 60 years ago. Sir Edward has been gravely misled into attributing our knowledge of canine hysteria to American sources of the past 30 years.

I find further support for my inference that Sir Edward's dogs were suffering from the B.H.S. and roundworm infections from his description of "the actual observed state of the affected animals" in such phrases as: "the attacks seem to represent temporary exacerbations superimposed on a chronic abnormal condition"; "in young puppies the attacks came on insidiously and usually took some weeks to develop"; "lactating bitches appear to be particularly prone to hysterical fits." I accept Sir Edward's diagnosis of his dogs' behaviour as canine hysteria, not his startling notion of its aetiology. In my opinion he experimented on diseased dogs and mistook the expressions of their disease for the consequences of his dietary changes. - I am, etc.

TOM HARE. REFERENCES

Eyre, J. W. H. (1928). Vet. J., 84, 183. (1938).' Vet. Rec., 50, 213, and Proc. N.V.M.A. Congress, (1946). Ibid, 58, 177

\section{Arsenical Encephalopathy}

SIR,-The report of a case of arsenical encephalopathy and its treatment, by Drs. G. Hipps and R. Goldberg (March 8, p. 296), calls for some comment. Though a case of this nature may be rare in this country it can by no means be described as " a rare clinical condition." Such cases were commonly met with in India Command in the years 1942-5, and a large number of deaths occurred from post-arsenical encephalopathy during that period. The condition was almost exclusively confined to Indian O.R.s, the majority being natives of Southern India. Though the diet of Indian troops varied a good deal in its constituents from that of British O.R.s, the part played by subclinical vitamin deficiency or by deficient intake of aminoacids containing the essential - SH grouping could not be determined in these fatal cases.

Though symptomatic treatment of effects due to organoarsenical intoxication is an important feature in the later stages of the syndrome, it is now known that specific treatment of the underlying chemo-pathology at an early stage will produce positive results. Recent work on the toxicity of arsenical compounds when injected into the body confirms the work of Voegtlin and his colleagues (Voegtlin, Dyer, and Leonard, 1923, 1925), who showed that organic compounds of the type R.As: As.R were inactive in that form but were oxidized in the body to the form R.As: $O$, and that only when they had undergone this change did they exert their toxic action either on the parasites or the host. They showed further that the 
arsenoxide so formed reacted with sulphydryl groups in the body tissues and in the spirochaetes. Hogan and Eagle (1944) showed that the toxicity of any arsenoxide depends on its constitution and its ability to combine with essential functional groups in vital organs.

Many of the enzymes concerned with carbohydrate metabolism are known to be inhibited by reagents which react with sulphydryl groups (Cori, 1946), and detailed work on this subject has been carried out by Barron and Singer (1945) using a variety of reagents. Glutathione is one of the most important substances in the body containing the thiol group. It is probable that the vitamin-protein enzymes are similarly adversely affected by organo-arsenicals, for it is known that arsenic interferes with the conversion in the liver of vitamin $B_{1}$ to its co-enzyme form. The poisoning action of arsenoxide on glutathione is probably one which involves oxidation of its thiol group to a disulphide, resulting in the production of oxidized glutathione. This in turn will bring about the inactivation of succinic dehydrogenase, and as glutathione acts as a catalyst for glyoxalase, the action of this latter enzyme will be also in abeyance. Damage even more widespread will follow; the presence of the sulphydryl group in several oxidizing enzymes, as well as in several proteolytic and other enzymes, has been shown to be necessary for their activity (Bersin, 1935; Hellerman, 1937; Barron and Singer, 1945). In view of the fact that oxidizing agents in the cell tend to inhibit the activity of $-\mathrm{SH}$ enzymes, it is probable that one of the main functions of glutathione in cellular systems is concerned with the continuous reactivation of the $-\mathrm{SH}$ enzymes. Its capacity to bring about such a reactivation through its powerful reducing action has been demonstrated in many experiments.

Once enzymic balance has been upset recovery is very possibly hard to effect, and reactions will proceed in one direction faster than metabolism can control them: Lactic acid synthesis is retarded to such a degree that enzymic control in other directions is affected by the building up of aldehydic compounds. It is postulated that this is due to the inactivation of glyoxalase and succinic dehydrogenase, with a subsequent block in the tricarboxylic acid cycle.

The following chain of events léading to the development of arsenical encephalopathy has been suggested by Lydon: (1) Interference with cellular metabolism. (2) Accumulation of metabolites in C.N.S. cells (which are most sensitive to changes in oxygen tension). (3) Increased retention of fluid by cells, with oedema of tissues. (4) Interference with normal capillary circulation. (5) Congestion and subsequent focal necrosis. The characteristic pathological changes found in fatal cases support this theory.

The immediate treatment of post-arsenical encephalopathy should be one which aims at preventing the establishment of the vicious circle mentioned above and opening the way for the restoration of the metabolic sequence known as the Krebs tricarboxylic acid cycle. . While it has been shown that the toxic action of arsenoxide is inhibited by the intravenous administration of reduced glutathione (Voegtlin et al., 1925), recent studies have proved that the most effective compound in use as an antidote against heavy metal poisoning is 2,3-dimercaptopropanol (BAL), which in dogs is many times more effective than glutathione (Long and Farah, 1946). It is recognized that these compounds exert their maximal therapeutic action when given at the earliest possible opportunity after a diagnosis of organo-metallic intoxication has been made. As excess of succinate protects its dehydrogenase from the irnhibitory effect of oxidized glutathione, the combination of a succinate and an organic thiol compound given parenterally should, in theory, give the maximal therapeutic result if administered immediately arsenical intoxication is suspected.

The practice of using intravenous glucose does not appear to be physiologically sound in view of the fact that a disturbance of carbohydrate metabolism is occurring. Double-strength plasma would seem to be the agent of choice in counteracting cerebral oedema. The use of a compound such as calcium thiosulphate does not present an available thiol radicle but merely introduces sulphur in inorganic form. One of the organic thiol or di-thiol compounds mentioned above will produce a far greater and more rapid therapeutic effect. These compounds are now quite easily obtainable.-I am, etc.,

Lancaster.

$$
\text { E. A. J. BYRne. }
$$

\section{REFERENCES}

Barron, E. S. G., and Singer, T. P. (1945). J. biol. Chem., 157, 221

Bersin, T. (1935). Ergebn. EnzymForsch., 4, 68.

Cori, C. F., and Cori, G. T. (1946). Ann. Rev. Biochem., 15, 193.

Hellerman, L. (1937). Physiol. Rev., 17, 454

Hogan, R. B., and Eagle, H. (1944). Ibid., 81, 142.

Long, W. K., and Farah, A. (1946). Science, 104, 220.

'Voegtlin, C., Dyer, H. A., and Leonard, C. S. (1923). Publ. Hlth. Rep., Wash., 38,1882

et al. (1925). J. Pharmacol., 25, 297.

\section{Treatment of Post-operative Pulmonary Atelectasis}

SIR,-The annotation on a new treatment for post-operative atelectasis (March 22, p. 386) prompts me to inform you of a method of dealing with this condition which I have used occasionally during the past two years. The method is to stimulate coughing by the introduction of $1 \mathrm{ml}$. of water, by means of a syringe, into the respiratory passages, the needle being passed into the trachea at about the level of the first ring - that is, below the cricoid cartilage. This method is not intended to supplant bronchoscopic aspiration, which, all observers agree, is the ideal method of treatment, and which should be undertaken as soon as signs of atelectasis appear, but the introduction of water may serve as a simple substitute if the facilities for bronchoscopic aspiration are not available.

The last case which I dealt with by this means was a man of 44 who had had a Polya gastrectomy for duodenal ulcer on March 4, 1947. When I visited him on the evening of March 5, he had an atelectasis of the left lower lobe. The surgeon who had operated on the case had left instructions that he was not to be disturbed, and, as he was not to be found, I decided to employ the water technique, though I should have preferred to use the bronchoscope. The effect was excellent: after a violent spasm of coughing, in which considerable sputum was brought up, he stated that he felt better, and his dyspnoea disappeared at once. He continued to bring up sputum for a few days, but all signs of atelectasis had gone, and the temperature, which had been raised on the evening of March 5 , became and remained normal.

The birth of this method occurred when I was anaesthetist to a surgical team for chest surgery in the B.L.A., the surgeon in charge being Major J. Leigh Collis, of Birmingham. A patient was brought in with collapse of the right lower lobe, the cause being a piece of tinned bacon which he had inhaled. I anaesthetized the patient for bronchoscopy by swabbing the pyriform fossae with $15 \%$ cocaine, and then introduced $1 \mathrm{ml}$. of the same fluid into the trachea through the top ring. The patient immediately coughed and expelled the greater part of the inhaled bacon. This suggested to Major Collis and myself the method of causing expulsive coughing which I have described above.

I am tempted to make two comments on Grandstaff's work to which your annotation was directed. First, although I have not seen the original article, I find great difficulty in believing that, of a total of $2.6 \%$ of respiratory complications following operation (an extremely low figure), two-thirds had pneumonia. My own experience, in common with everyone else whom I have consulted, shows that atelectasis is by far the commonest of major respiratory post-operative complications, and that the pneumonia rate is only a very small fraction of this. Secondly, while not denying the possibility, remote though it appears to be, that analgesia of the larynx may cause dilatation of the bronchi, it would hardly appear that this of itself would cure atelectasis: it is also necessary that the offending plug be removed, either by aspiration or by coughing. With all due deference to the author of your annotation, swabbing of the pyriform fossae does not "inevitably produce severe coughing"; in fact, it practically never does. For this reason Grandstaff's method might well be improved by stimulation of coughing by some such means as I suggest.

While on the subject, I should like to mention a point of diagnosis which $I$ have never seen in print: the dyspnoea in atelectasis is always more severe than the physical signs would appear to warrant, and the sudden onset of severe dyspnoea after operation is strong presumptive evidence of atelectasis.

It is a very encouraging sign that attention is now being directed to the diagnosis and treatment of post-operative atelectasis. Lives have been, saved, and many days of convalescence averted, by the prompt use of the bronchoscope. It is most necessary that all anaesthetists should become expert in the use of this instrument, and also expert diagnosticians of thoracic disease. Too often, even now, surgeons are content to classify all cases of atelectasis, bronchitis, pneumonia, or what-have-you as "anaesthetic chests" and to bother no more about them

In conclusion, while stressing the need for early bronchoscopic aspiration, let it not be forgotten that postural coughing, 